\title{
The origin of the multiple stellar arcs
}

\author{
Yu. N. Efremov \\ Sternberg Astronomical Institute, MSU, Moscow 119899
}

\begin{abstract}
We argue that multiple stellar arcs are formed by single beamed super-explosions or long-standing jets produced by objects connected with Gamma-ray bursts.
\end{abstract}

\section{Introduction}

There are giant arcs of luminous stars and young clusters in the region of the supershell LMC4 in the LMC. The most obvious was noted by Westerlund and Mathewson (1966), who ascribed the origin of the arc to a super-Supernova explosion, referring to the paper by Shklovsky (1960). This arc and two others in the same region were sketched by Hodge (1967), who also found similar arcs in NGC 6946, recently confirmed by picture given by Larsen and Richtler (1999).

The multiple arcs in the LMC contains young clusters of about the same age (Efremov and Elmegreen, 1998) as do the arcs in NGC 6946 (Efremov, Elmegreen, Larsen and Richtler, 1999). The strictly circular shape, giant sizes $(150-300 \mathrm{pc}$ radius) and coeval ages prove beyond any doubt the coherent origin of objects in arcs. The positions of various features in the LMC4 region are shown in Figure 1 in Efremov and Elmegreen (1998) and in Figure in Efremov (1999a).

\section{The multiple arcs in the LMC}

Efremov and Elmegreen (1998) suggested that two well-shaped arcs in this region were formed by triggered star formation in gas, swept-up by centralized sources of pressure. The strictly circular shapes of both arcs are the strongest evidence for this. The small clusters near centers of both arcs were suggested to contain earlier O-star and $\mathrm{SNe}$ which were sources of the pressure.

These centralized stellar sources of pressure could produce both young stellar arcs at the right time and position, as Efremov and Elmegreen (1998) demonstrated, yet the general picture is still not satisfactory. The main questions remaining are: (1) why are there no giant stellar arcs or rings around other, even much more rich, clusters in the LMC, (2) why are all of the stellar arcs in the LMC close to each other and why are they only in this area, (3) why one arc is inside the LMC4 supershell instead being at its rim, and inside the smaller arc there is gas which must have been swept-up, (4) why are there just arcs and not full stellar rings, and (5) why are these arcs in the region of the largest and deepest $\mathrm{H}$ I hole in the LMC? 
Indeed, what was so special with these small clusters to produce unique arcs? There is increasing evidence for a common absence within the $\mathrm{HI}$ supershells of clusters rich enough to contain (in the past) sufficiently numerous $\mathrm{O}$-stars and $\mathrm{SNe}$ to trigger the formation of supershells. Recently Rhode et al. (1999) found that only within 6 of the 44 supershells in Ho II there are clusters which could contain $\mathrm{SN} / \mathrm{O}$ stars numerous enough to form the presently observed supershells.

The recent identification of GRB afterglows in distant galaxies has revived the possibility that single super-explosions do exist. It was shown that they can produce very large shells and trigger star formation (Blinnikov and Postnov, 1998; Efremov, Elmegreen and Hodge, 1998; Loeb and Perna, 1998; Efremov, Ehlerova and Palous, 1999). This suggestion explained the enigmatic supergiant H I shells without a central cluster or evidence of an extragalactic cloud impact in the triggered region.

One could imagine that the oblique infall of a group of a few clouds could produce these 3 or 4 arcs in the LMC, considering especially that the orientation of two arcs is rather similar. This could probably explain that the arcs are not full rings. However, the age difference between the clusters in arcs is large enough (Efremov and Elmegreen, 1998) that one has to assume that is was a kind of a stream of clouds along a similar orbit. Furthermore, given that the LMC rotates, the infall of all clouds just near each other seems to be improbable.

\section{The arcs from GRB hypothesis}

We conclude the that most probable sources of the central pressure which formed the arcs in the LMC were the energetic super-explosions. The only known objects with which such events is possible to identify, are Gamma-ray bursters. This explains the absence of evident clusters in the centers of arcs yet why would all the peculiar outbursts occur in the same region of the LMC? The only explanation is that the progenitors of these outbursts were the individual stars or binary systems which escaped from a massive near-by cluster (Efremov 1999a, $1995 \mathrm{~b})$.

The common source of the GRB progenitors must have been somewhere in the same region of the LMC. These progenitors are close binaries with neutron stars (as X-ray binaries are) and merging in such a binary give the GRB event. There should be a dense massive cluster where such stars have formed and then escaped. There is indeed such a cluster in the region under consideration - NGC 1978 is within $1-1.5 \mathrm{kpc}$ of both arcs and the center of the LMC4 supershell. The age of this cluster is about $2 \mathrm{Gyr}$ (Bomans et al. 1995), and it is the richest cluster of such an age in the LMC. Its mass is $(0.4-1.4) \times 10^{6} M_{\odot}$ (Meylan et al. 1991) and it has a few hundred red giants with masses of around $1.5 M_{\odot}$.

The high rate of occurrence of X-ray binaries inside dense globular clusters is well known (e.g. Bailyn 1996). It was explained long ago as consequence of the high probability of formation of close binaries after tidal captures in the dense cluster (e.g. Fabian et al. 1975, Shklovsky 1982, Davies 1995, Phinney 1996). It was also shown (McMillan 1986) that a large number of tidally captured binaries may escape a dense old cluster as the result of three-body encounters. The comprehensive review of the data on binaries and pulsars in globular clusters 
(Phinney 1996) left no doubts that there is a lot of possibilities to form close binaries with compact components in a cluster dense and old enough, and also that many of these binaries are able to escape from such a cluster.

There is also a special way for binaries with a neutron star component (merging in such systems leads to a GRB event) to escape from a cluster. The formation of a neutron star after a SN explosion in a binary system leads to a high kick velocity, the most likely value of which is $150-200 \mathrm{~km} \mathrm{~s}^{-1}$. Even much smaller velocities would disperse the GRB progenitors significantly, because the binaries may take $100 \mathrm{Myr}$ or so before they merge to give a GRB (Lipunov et al. 1997, Bloom et al., 1999).

NGC 1978 is also unusual in its extremely flattened shape (Geisler and Hodge, 1980). This may indicate a formation process involving the merger of two clusters, especially because no rotation has been detected (Fisher et al. 1992), or owing to disk-shocking and might point to the dynamical state of the cluster which permits the escape of stars irrespective of their masses - and therefore, binary stars as well.

Many binary systems could escape from this massive cluster and among them there could be progenitors of GRB. Hanson and Murali (1998) suggested that stellar encounters in globular clusters were able to produce not only millisecond pulsars but also binaries that evolve into GRBs. Also, Spruit (1998) suggested a way to make a GRB from an X-ray binary connected with accretion to differentially rotating magnetic neutron stars. In his scheme the millisecond pulsars are the remnants of X-ray binaries that managed to escape from the fate to evolve to GRB.

Both X-ray binaries and millisecond pulsars are indeed found in the region of NGC 1978. Near the cluster there is excess number of X-ray binary stars, three X-ray binaries are within 20' of NGC 1978 and more are in a wider surrounding area, as is evident from Haberl and Pietsch (1999). About half of X-ray binaries known in the LMC are within a 3 square degree area near LMC4 and NGC 1978. In the same area and close to NGC 1978 is also the object which is even more certainly related to GRB. It is the the soft gamma repeater (SGR), SGR0526-66, which produced the famous gamma-burst of March 5, 1979 and is within N49, the brightest SNR in the LMC. With this SGR, three or four stellar arcs and supershell LMC4, we have five or six GRB events in the LMC during the last $30 \mathrm{Myr}$ or so, which seems to be a quite reasonable amount.

The only other known system of multiple arcs was noted by Hodge (1967) in the spiral galaxy NGC 6946. Recently Larsen and Richtler (1999) independently found Hodge's multiple arcs in NGC 6946, describing these as a spherical bubble of clusters with a diameter of $550 \mathrm{pc}$. Moreover, the diffuse object inside the arc system was proved to be the most massive young cluster in the galaxy, with $M_{V}=-13$. Thus, near each of both known systems of multiple arcs, in the LMC and in NGC 6946 there are very massive clusters.

The bubble of arcs and the large young cluster in NGC 6946 is a unique object: nothing similar was found in the sample of 21 galaxies studied by Larsen and Richtler (1999). Its nature is really enigmatic. It would be possible that these arcs were formed by massive stars (which are suggested to be able to outburst as Hypernovae to give a GRB-like event) escaped from the young massive cluster while it was still at the stage of the violent relaxation. However, the pre- 
liminary ages of that cluster and of the smaller clusters in the arcs are similar. There is also another possibility: the arcs cross at the position of the globular and if there was a collision of gas shells, this could lead to the formation of a massive bound cluster (Efremov, Elmegreen, Larsen and Ricthler, 1999). In this case, another older cluster, from which the progenitors of arcs might have been ejected, should be somewhere nearby.

\section{Discussion}

The properties of stellar arcs, suggested here to be GRB relics, may say something on the nature of the GRB event. First of all this is surely the opening angle of the arcs, which is always smaller then 90 degrees. Another important property of the arcs is their wonderfully strict circular shape, independent of the galaxy plane inclination. This implies the arcs are partial stellar lined spheres, seen in projection, and not rings in the plane of galaxy. Even an isotropic outburst could result in a partial gas shell, if exploded outside a galaxy gas disk. Owing to the density gradient, the most dense part of a swept-up shell is then turned to or off the galaxy line of nodes and looks like an arc (Efremov, Ehlerova and Palous, 1999). However, this is not the case for the arcs in the LMC. Therefore, the arcs were not formed from isotropic outbursts outside the gas disk of the galaxy. The very special position of the parent outburst inside the large gas cloud, yet in an excentric position, is another way to explain the existence of stellar arcs and their orientation (Efremov et al., 1999). The only possibility to avoid such an ad hoc assumption is to assume that the opening angle of the arcs reflects the opening angle of the parent collimated radiation or explosion. Considering many arguments exist for very narrow relativistic jets from the GRB (e.g. Fargion, 1998; Dar, 1999; Kulkarni et al. 1999; Sari et.al., 1999) the conclusion seems to be unavoidable that these narrow jets were long ejected under variable angles within the opening angles of stellar arcs.

Thus we come to the necessity to admit long-standing precessing jets with variable angle of precession. The idea of such spinning and multi-precessing narrow jets has already been advanced as an explanation of GRB events but from completely other considerations (Fargion, 1998, 1999).

The mechanism of star formation under action of such jets should be investigated. The impact of an energetic jet onto a gas cloud could trigger its collapse and star formation, yet if the jets triggered star formation in pre-existing clouds, the very regular shape of stellar arcs is difficult to explain. The long standing jets are probably able to sweep up the gas into partial shells and variable angle of precession could lead to formation of filled partial shells. This could explain some of points (1) - (5) above.

The star formation induced by long-acting precessing jets from the active galactic nuclei is really observed. The nearest example of such event is found in Cen A galaxy (Graham, 1998; Morganti et al. 1999).

Acknowledgments. I am grateful to the IAU for a grant permitting participation in the Colloquium and to the astronomers of Turku for their hospitality. 


\section{References}

Blinnikov, S. I. and Postnov, K. A., 1998, MNRAS, 293, L29

Bailyn C. D. 1996, ASP Conf. Ser., 90, 320

Blackman E. G., Yi, I. and Field, G. B. 1996, ApJ, 473, L79

Bloom, J. S., Sigurdsson, S. and Pols, O. R., 1999, MNRAS, 305, 763

Bomans, D. J., Vallenari, A. and de Boer, K. S. 1995, A\&A, 298, 427

Dar, A. 1999. Preprint astro-ph/9905315

Davies, M. 1995, MNRAS, 276, 887

Efremov, Yu. N. 1999a, Astron. Lett. 25, 74

Efremov, Yu. N. 1999b, Astron. Rep. 43, 284

Efremov, Yu. N. and Elmegreen, B. G. 1998, MNRAS, 299, 643

Efremov, Yu. N., Elmegreen, B. G. and Hodge, P. W. 1998, ApJ Lett., 501, L163

Efremov, Yu. N., Ehlerova, S. and Palous, J., 1999, A\&A, accepted

Efremov, Yu. N., Elmegreen B., Larsen, S. and Richtler, T. 1999, ApJ Lett., submitted

Fabian A. C., Pringle J. E. and Rees, M. J. 1975, MNRAS, 172, 15P.

Fargion, D. 1998, astro-ph/9808005

Fargion, D. 1999, astro-ph/9906432

Fisher, P., Welch, D. L. and Mateo. M. 1992, AJ, 104, 1086

Geisler, D. and Hodge, P. 1980, ApJ, 242, 66

Graham, J. A., 1998, ApJ, 502, 245

Haberl, F. and Pietsch W., 1999, A\&A, 344, 521

Hanson B. M. S. and Murali, C. 1998, ApJ Lett., 505, L15

Hodge, P. W. 1967, PASP, 79, 29

Kulkarni, S. R. et al. 1999. preprint astro-ph/9902272 (submitted to Nature)

Larsen S. S. and Richtler, T., 1999, A\&A, 345, 59

Loeb A. and Perna R. 1998, ApJ Lett., 503, L35

Lipunov, V. M., Postnov, K. A. and Prokhorov, M. E. 1997, MNRAS, 288, 245

MacMillan S. L. 1986, ApJ, 307, 126

Meylan, G., Dubath, P. and Mayor, M. 1991, In: The formation and evolution of star clusters, p. 158

Morganti, R., Killeen, N. E. B., Ekers, R. D. and Osterloo, T. A., 1999, preprint astro-ph/9903448

Phinney, E. S., 1996, ASP Conf. Ser., 90, 163

Pugliese, G., Falcke, H. and Biermann, P. L., 1999, A\&A, 344, L37

Rhode, K. L., Salzer, J. J., Westfahl, D. J. and Radice, L. A., 1999, AJ, 118, 323

Shevalier, R. A. and Li, Z.-Y., 1999, Preprint astro-ph/9904417

Spruit H. C. 1999, A\&A, 341, L1

Shklovsky I. S. 1960, Soviet Astron. - AJ, 4, 355

Shklovsky I. S. 1982, Comments on Astrophys., 9, 261 
Westerlund, B. E., and Mathewson, D.S. 1966, MNRAS, 131, 371 